\title{
Decreasing Memory Overhead in Hard Real-Time Garbage Collection*
}

\author{
Tobias Ritzau and Peter Fritzson \\ Department of Computer and Information Science \\ Linköping University, SE-581 83 Linköping, Sweden \\ \{tobri,petfr\}@ida.liu.se
}

\begin{abstract}
Automatic memory management techniques eliminate many programming errors that are both hard to find and to correct. However, these techniques are not yet used in embedded systems with hard realtime applications. The reason is that current methods for automatic memory management have a number of drawbacks. The two major ones are: (1) not being able to always guarantee short real-time deadlines and (2) using large amounts of extra memory. Memory is usually a scarce resource in embedded applications.

In this paper we present a new technique, Real-Time Reference Counting (RTRC) that overcomes the current problems and makes automatic memory management attractive also for hard real-time applications. The main contribution of RTRC is that often all memory can be used to store live objects. This should be compared to a memory overhead of about $500 \%$ for garbage collectors based on copying techniques and about $50 \%$ for garbage collectors based on mark-and-sweep techniques.
\end{abstract}

\section{Introduction}

Memory related errors are often very hard to find and correct, since they may be located at places different from where they appear. For instance freeing the same memory region twice may not cause a problem until the region is reused. Automatic memory management, also referred to as garbage collection or GC, is used to reclaim memory that is no longer used by a computer program. This greatly reduces the risk of memory related errors in programs.

Tracing garbage collectors, e.g. copying, mark-sweep, and threadmill collectors, all share the problem that unreachable (garbage) objects are not reclaimed immediately when they become unreachable. This dramatically increases the memory overhead. Most tracing real-time GCs perform an increment of GC work when memory is allocated. The amount of work $(W)$ performed is proportional to the amount of memory that is allocated $(A)$. In short, the amount of work needed should be enough to complete a full GC cycle (to collect all unreachable objects) before all memory overhead $(O)$ is used up by new objects.

\footnotetext{
* Supported by the EC funded IST project HIDOORS (High Integrity Distributed Object-Oriented Realtime Systems) and the ESA funded AERO project (Architecture for Enhanced Reprogrammability and Operability).
} 
The memory overhead is used as a buffer to store new objects while unreachable objects are collected. To calculate $\mathrm{W}$, the total amount of GC work needed to complete a full cycle must be known $(G C W)$. The work needed can be calculated as $W=G C W \times A / O$. Thus, the execution time is inversely proportional to the amount of memory overhead in the system. In a typical two-subheap copying collector $500 \%$ memory overhead is needed and in a typical mark-sweep collector $50 \%$ is needed. By using the technique presented in this paper no memory overhead is needed to store new objects. This is of extremely valuable in embedded systems with scarce memory resources.

Reference counting 9] has been known to have a number of disadvantages, causing many implementers to avoid its use. However, reference counting also has numerous advantages, especially for use in embedded systems.

The idea of reference counting is simple: count the number of references to every object and recycle the object if its reference count becomes zero. A reference counting memory handler contains two main operations: increment and decrement of reference counters. The decrement operation also frees objects when their reference counters become zero.

The advantages of reference counting are its simplicity, memory is immediately reclaimed when it becomes unreachable, fine-grainedness (only a few instructions need locking in multi-threaded systems), it does not use separate garbage collection code to reclaim memory (all work is done by the increment and decrement operations), and that data is not moved back and forth between different memory regions.

Simplicity makes reference counting more feasible in safety critical systems, since the method is easier to implement and to prove correct. Because of its fine-grainedness, reference counting is suitable for multi-threading, especially for uniprocessor systems where locking is cheap. Since all updates are performed by the increment and decrement operations, there is no need to execute garbage collection code when allocating memory or at any time other than when performing reference count updates. This simplifies worst-case execution time analysis. Reference counting keeps data in fixed memory locations, in contrast to copying garbage collection techniques. Keeping data at fixed locations simplifies interfacing to other systems and languages. Copying data also requires more memory, takes extra time, and makes synchronization between the garbage collector and the program harder.

However, there are also some disadvantages with standard reference counting. Recursive freeing occurs when the last reference to a large data structure is removed. This may cause long interruptions that may be hard to predict. Since memory is not compacted, fragmentation may also be an issue. In most applications fragmentation causes no problems, but it may in a safety critical system. Reference counting is often considered to be slow, and the basic technique is. The inability of reclaiming cyclic data structures is often considered the major drawback of reference counting.

Recursive freeing can be eliminated using a technique described by Weizenbaum [19]. The decrement operation is changed to put the object into a list 
instead of decrementing its children and freeing it (see Fig. 1). If the system runs out of memory when allocating an object, the list is processed and dead memory is reclaimed. This solves the problem of recursive freeing, but on the other hand garbage collection code needs to be executed when allocating objects. The technique is designed for applications where all objects are of the same size, but can easily be adapted to suit other systems as well.

Dividing objects into blocks of equal size can be used to handle external fragmentation. This approach is further discussed below.

Several techniques to reduce the execution time overhead of reference counting have been proposed. All of these aims to reduce the number of reference count updates either by not counting all references [102] or by statically finding redundant reference count updates 3 .

Cyclic data structures can be reclaimed using several techniques. These include weak pointers [6], partial mark-sweep [8,1], reference counting cyclic data structures instead of their components 4 and using a backup real-time garbage collector. Partial mark-sweep algorithms require no manual administration, but the others do. This can cause problems since people are known to make mistakes, so they should be used with caution in safety critical systems. Partial mark-sweep techniques are feasible in interactive systems, but have not been proven to work in hard real-time systems.

This paper presents a technique that does not suffer from external fragmentation and is fully predictable in both memory usage and execution time. The main advantage is that the worst-case execution time of GC work is improved from $O(s+1 / f)$ to $O(s)$, where $s$ is the size of the object and $f$ is the minimum amount of free memory (the overhead) in many systems. Since the minimum amount of free memory is not a factor, all memory can be used for live objects. This is extremely valuable, especially in embedded systems, where memory usually is a scarce resource.

We classify real-time systems into three categories: interactive, soft real-time and hard real-time. Interactive systems are systems that interact on-line with the external world, e.g. X-Windows. There are not any deadlines on the response on these systems, but too long response times are annoying to the user. Soft realtime systems have deadlines but slightly missing one now and again does not cause any problems. Multimedia applications are examples of soft real-time. In hard real-time systems a deadline should never be missed. Missing a deadline could cause disaster. Medical equipment and on-board aircraft control systems are examples of hard real-time systems.

The term "real-time garbage collection" is often used even for interactive applications. However, this is not the target of our research. The technique presented in this paper targets the embedded hard real-time systems.

In this paper everything that is allocated on the heap is called an object. A dead object is an object that will not be used by the program anymore. The children of an object are the objects that are referred to by the object.

The remainder of this paper is organized as follows. Section 2 proposes a reference counting technique for hard real-time systems. In Section 3 a prototype 
implementation is presented. Section 4 presents an evaluation. Section 5 presents related work and Section 6 presents the conclusion and future work.

\section{Real-Time Reference Counting}

The following sections describe how the disadvantages of the standard reference counter can be eliminated.

\subsection{Eliminating External Fragmentation}

In most systems fragmentation causes no problem [13], but in hard real-time systems it must be guaranteed that fragmentation does not cause the system to run out of memory. Fragmentation can be eliminated by compacting memory, e.g. using copying [1] or mark-compact 15 garbage collection. However, these methods are often considered too expensive and/or unpredictable. Other solutions include prohibiting heap allocation or limiting allocation into arenas, large memory areas that are freed as a whole.

The technique proposed in this paper is based on dividing objects into equally sized blocks, as applied also in many file systems and virtual memory systems. This eliminates external fragmentation, but introduces internal fragmentation, i.e. the last block of an object will not always be fully used. An important difference between internal and external fragmentation is that internal fragmentation is predictable, but external is not.

There are several ways to connect blocks into an object, e.g. linked list 17 and index blocks. In the rest of this paper we assume that the blocks are kept in a linked list, but other techniques work as well. The requirements are that member access should be predictable, that taking a step in the iteration through the blocks of an object should take constant time, and that it must be possible to disconnect a block from the object in constant time.

\subsection{Eliminating Recursive Freeing}

The increment operation of reference counting just increments a variable, an operation with predictable worst-case execution time. However, the decrement operation is potentially recursive. In the worst case a decrement operation can free all objects on the heap, and even if the size of the heap is bounded, it is too long for essentially all real-time applications.

By using deferred reference counting 19] the decrement operation becomes constant in time. Using deferred reference counting, objects are added to a tobe-free list instead of decrementing its child references and freeing the object (see Fig. 1.) The child references still need to be decremented, which now is done by the allocator instead. When the allocator needs more memory, objects are taken from the to-be-free list. Before they are reused their child references are decremented. 


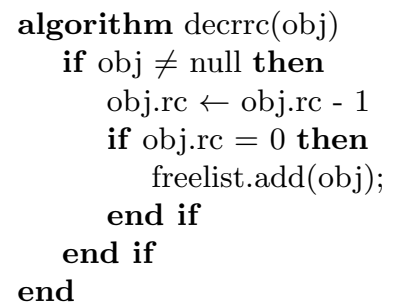

Fig. 1. Deferred reference counting

Deferred reference counting assumes that all objects are of equal size, but that is not case in most systems. The technique can be applied using RTRC since all blocks are of equal size, even though objects can contain multiple blocks. Using the deferred reference counting, all child references are decremented when an object is taken from the to-be-free list. This could cause problems when freeing large objects. However, if only the blocks that used by the new object are processed, the execution time becomes proportional to the size of the allocated object and does not depend on the objects in the to-be-free list. It must be possible to get a single block from the free list in constant time for this to work.

\subsection{Reclaiming Cycles}

Cyclic data structures are reclaimed using a real-time mark-sweep garbage collector. This increases the execution time overhead, but since the reference counter has loaded the objects into the cache, the overhead is small. No extra memory overhead is needed since the marks-sweep flags could be stored in the reference counter field (since the reference counter does not need all bits). The effect of combining these techniques is that all dead memory is reclaimed with drastically lower memory overhead (compared to real-time mark-sweep). The execution time of a real-time mark-sweep collector is proportional to $1 /$ memory overhead. Practically, this means that about $50 \%$ memory overhead is needed to get acceptable execution time. The execution time of the combination is decreased by the ratio of cyclic and non-cyclic garbage. This factor could by anything from 0 (no cycles) to 1 (all garbage belong to cycles). Many systems can be designed to produce little cyclic garbage.

If cycles can be guaranteed to be completely eliminated manually, e.g. using manual cycle breaking and/or weak pointers, no mark-sweep collector is needed. Thus, all memory could be used to store live blocks.

We now have achieved a system without external fragmentation and with predictable allocation and reference count update operations. It is also guaranteed that all dead blocks that do not belong in cycles are immediately available to new objects. Thus, memory overhead decreases or is even eliminated completely. If it is desirable to increase the speed of allocation in high-priority processes [12] this can be achieved by forcing the free list (which contains blocks which child refer- 
ences have already been decremented) to contain the number of blocks needed by the high priority process.

Dividing objects into blocks introduces an overhead in both memory usage and execution time. Using a linked list schema, each object needs a pointer to the next block. These pointers need to be traversed when accessing data in blocks other than the first. The number of pointers to traverse to get a field is known at compile time. Large objects such as arrays should not use a linked schema, because that would make the execution time of an indexing operation a linear function of the size of its argument. Arrays can be stored as trees that make the execution time a logarithmic function of the size of the array. To get the best performance, arrays can be allocated statically and contiguous.

The execution time overhead of object access is comparable to that of realtime copying garbage collectors, which always use one indirection [5] to find the current copy of the object. Thus, the block size should be selected to fit most objects in one or two blocks. Two other factors that are important when selecting block size is internal fragmentation and the cache memory. Experiments have shown that 32 or 64 byte blocks give good results in many applications.

The best block size depends on the application. To tune the application, the allocation function can be used to produce statistics about how many objects of different sizes are allocated.

The only disadvantage that remains in this proposal is the execution time overhead. The execution time overhead could be improved by static optimizations, such as removing redundant reference count updates and allocating objects on the stack.

\section{Implementation}

The RTRC technique has been implemented in the JOSES Java ${ }^{\mathrm{TM}}$ compiler, JoC 18 14. During compilation the object types are divided into records smaller then a specified block size. Then all member accesses are updated to handle divided objects. Finally the run-time system was updated both to access members in divided objects and to create and handle such objects. The implementation only supports the linked list schema. Arrays are currently not divided, so they can cause external fragmentation. We are planning to implement arrays as trees of blocks in the near future. There is also no backup garbage collector in the system. RTRC has also been implemented as a set of CPP macros for use with C.

The data layout of objects had to be changed to eliminate external fragmentation. An object is constructed of blocks. Each block has a next pointer, pointing to the next block in the object. RTRC also need type information, a reference counter and a pointer that links the objects when they reside in one of the free lists (the to-be-free or free list). These fields are needed in the first block of all objects. Since the reference counter is not needed when the object reside in a free list, the pointer and the reference counter can share memory. The memory overhead per object is 4 bytes for reference counter/free list pointer plus 4 bytes per used block. 
Two free lists are used by the run-time system. The to-be-free (tbf) list contains all objects that are dead, but their children have not been decremented, i.e. they have live references to the heap. The free list (freelist) keeps blocks that can be allocated immediately. A variable (available) keeps track of the number of blocks in the free list.

The run-time system is initiated by connecting all blocks on the heap into one free list. The worst case execution time of initialization is proportional to the number of blocks on the heap.

Allocating blocks from the free list is done by traversing the free list until the requested number of blocks has been found, terminating the list of blocks, and adjusting available and freelist. The worst case execution time of the function is proportional to the number of blocks being allocated.

Allocating from the to-be-free list is slightly more complicated. The function is shown in Fig. 2 and important data structures are shown in Fig. 3 First, one object is taken from the to-be-free list. The type information is stored in type; blockno is set to zero, and head points to the first block of the object that is allocated. For each block being allocated its child references are decremented using the decchildren function stored in the type information. If we run out of blocks in the current object, the next object in the to-be-free list is taken and its blocks are used. The execution time of function is proportional to the number of blocks being allocated.

The user function used to allocate objects allocates as many blocks from the free list as possible. The rest of the blocks are allocated from the to-be-free list. Finally, the lists of blocks are concatenated. The worst case execution time is proportional to the size of the object being allocated.

As stated earlier, it is sometimes beneficial to increase the execution speed of high-priority processes. In RTRC this can be accomplished by guaranteeing that all allocations of high-priority processes can be done from the free list. Thus, no time is spent decrementing child references. The run-time system contains a function (predec) which allocates blocks from the to-be-free list and put them directly into the free list. The allocation function must then always leave a specified number of blocks in the free list, except when allocating objects in high-priority processes. After the execution of high-priority code, the predec function must be called, so the free list is filled with blocks again. The number of blocks needed in the free list can be calculated using the technique presented in Henriksson's thesis [12]. The worst case execution time of predec is proportional to the number of blocks being pre-decremented.

The decrement and increment operations are implemented using deferred reference counting as presented in Fig. 1. The execution times of both operations are constant.

\subsection{Complexity and Overhead}

Initiating the memory manager is a matter of putting all blocks into a linked list. This could cause problems for virtual memory systems, but the embedded 


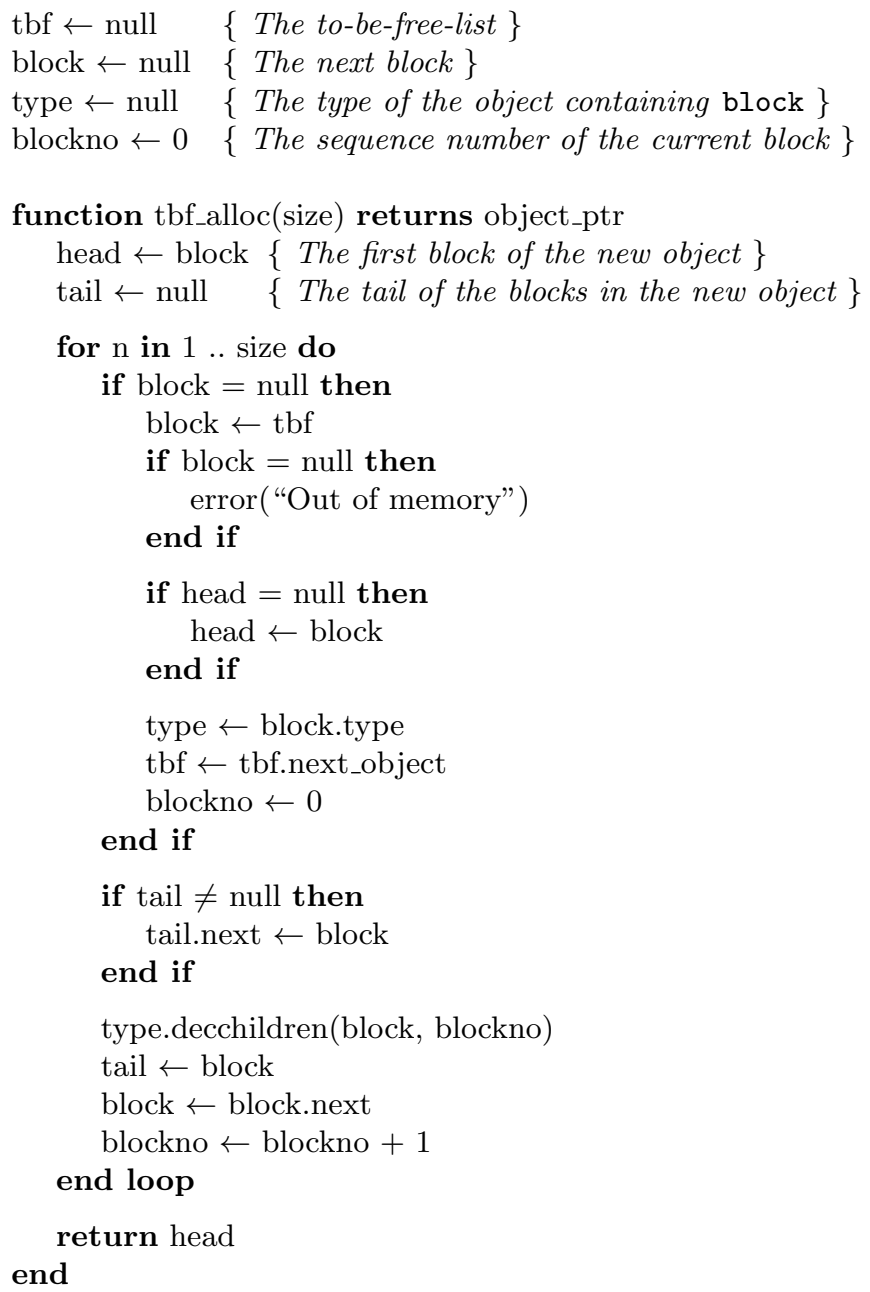

Fig. 2. Allocating blocks from the to-be-free-list

systems we target do not have virtual memory. The worst-case execution time is linear with respect to the size of the heap.

Allocation (from the to-be-free-list or free-list) takes blocks from the respective list. When blocks are taken from the to-be-free-list their references must also be released. Since releasing references of a block takes constant time (the size of blocks is constant and thus the maximum number of references), the worst-case execution time of an allocation is linear with respect to the size of the allocated object. If the system can produce dead cycles, a backup mark-sweep collector is used. Then, some mark-sweep work is done here as well. The amount of work is proportional to the amount of memory allocated. 

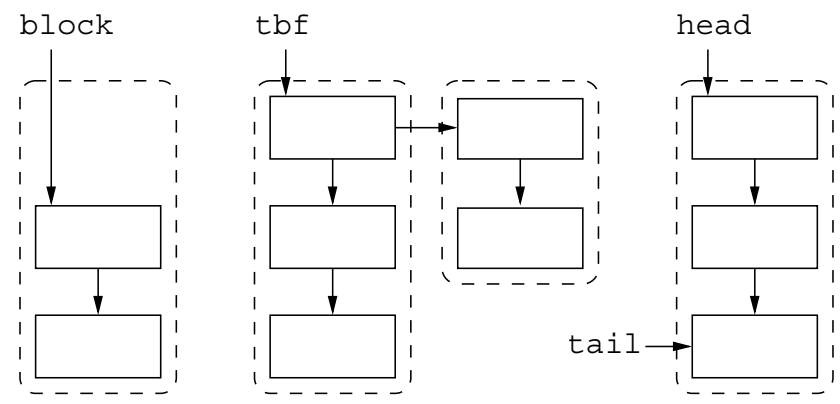

Fig. 3. Data structures used by tbf_alloc. The dashed boxes represent objects and the solid boxes represent blocks. To the left is the free object from which the blocks are currently taken. In the middle are the objects in the to-be-free list and to the right is the object being allocated.

In some systems it is desired that allocation in high-priority processes should be as fast as possible. This can be accomplished by pre-decrementing references of blocks in the to-be-free-list and moving these to the free-list. This is done by the predec function. The worst-case execution time of this function is proportional to the number of blocks that should be pre-decremented.

Finally, both the increment and decrement operations have a constant worstcase execution time.

\section{Timing Benchmarks}

All applications measured are compiled in various versions using RTRC, and without using any GC at all. The results presented are given as the execution time overhead in percent. Thus, a negative result is an improvement.

\subsection{Java Grande Benchmarks}

The RTRC technique has been evaluated using the Java Grande sequential benchmark 7]. These benchmarks were chosen by the JOSES project to evaluate the JoC compiler. Since the compiler is still unstable, we had to focus being able to compile one benchmark. The programs were compiled in four versions: without GC, with GC and contiguous, i.e. not divided, objects, with GC and 64-byte blocks, and with GC and 32-byte blocks.

Low Level Operations. The first section of the Java Grande sequential benchmarks contains tests of various low level operations, such as addition, multiplication, division and assignment. These where removed by the optimizer, except in one case that contained reference assignments. The test of reference assignments were optimized away when not using the reference counter, but not otherwise. However, such code should not appear in a real application. 
Kernels. Section 2 of the Java Grande sequential benchmarks contains various computational heavy applications, such as IDEA-encryption, FFT and matrix multiplication. These applications make little use of objects and therefore cause only a small amount of garbage collection work. The tests are performed in three variations with different size of the input. The relative overhead is similar in all variations. The average overhead is $10 \%, 4 \%$, and $11 \%$ for the contiguous, 64-byte, and 32-byte versions respectively. The results are presented in Fig. 4.

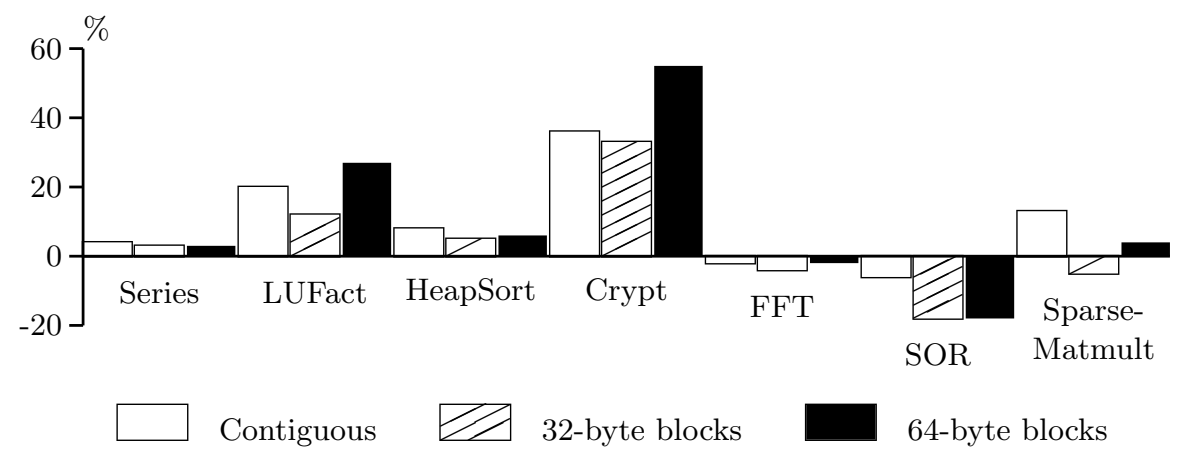

Fig. 4. Kernels benchmarks

Large Scale Applications. The JOSES compiler JoC had some difficulties compiling the applications in section 3 of the Java Grande Benchmarks. The applications are object-oriented and perform computational heavy calculations such as ray-tracing and alpha beta search. Only the alpha beta search binary could be executed with a valid result. The overhead in this test is significant, which can be explained by the amount of redundant reference count updates that is generated for all temporary reference variables that JoC generates. The overhead can be significantly reduced by static analysis. However, this is not yet implemented. The results show a $28 \%, 30 \%$ and $47 \%$ execution time overhead in the contiguous, 32 -byte and 64-byte versions respectively.

\subsection{Control System Application}

To measure the impact of different memory usage profiles on a more realistic hard real-time application, RTRC was also tested on a small simulation of a control system application. This test was written in $\mathrm{C}$ to get more control of the generated code. The control system was tested in 12 variations with and without: dividing objects in two blocks of 32 bytes each (d), separating the blocks of an object by half the heap size (ds), reference counting (r), and running a thousand simultaneous simulations ( $\mathrm{t}$ ). The result of this evaluation is presented in Fig. [5]

The conclusion from these tests is that dividing objects is expensive when using more memory than can fit into the cache. However, it should be noted that no reference counting optimizations are performed. By comparing the reference counted variations to their non-reference counted counterparts, we get a 


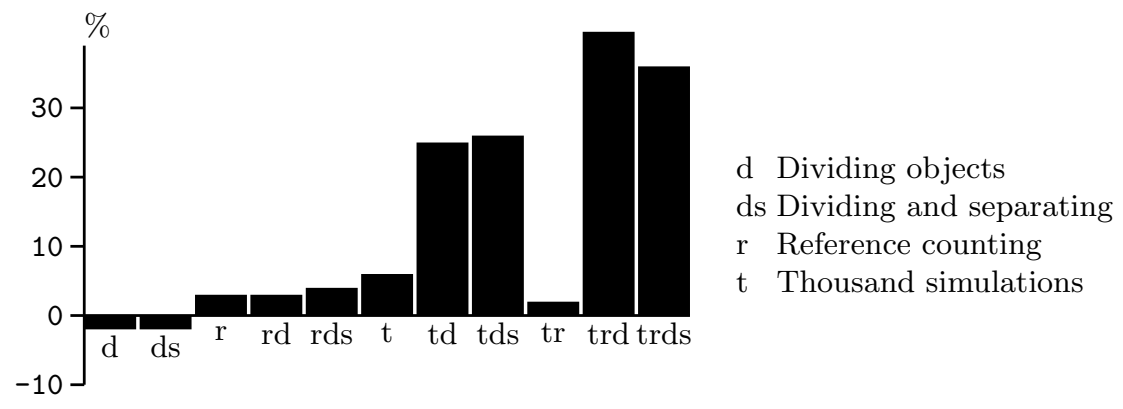

Fig. 5. Control system benchmarks

maximum overhead of $13 \%$. The lowest overhead was achieved when comparing the test with a thousand simulations without dividing objects to its reference counted counterpart. The reference counted version was $4 \%$ faster. Over 24 million increment and decrement operations were performed in each run of the system (with reference counting enabled).

\section{Related Work}

\subsection{Scheduling Garbage Collection}

Henriksson proposes a scheduling strategy that can be implemented together with many different garbage collectors. An implementation using an incremental copying garbage collector based on Brook's algorithm [5] is presented in Henriksson's thesis [12]. The processes are divided into high and low priority processes. The high priority processes do a minimal amount of garbage collection work to increase their speed. The main garbage collection work is instead performed when entering the low priority processes and during their execution. To guarantee that high priority processes do not run out of memory, enough memory must be preallocated. Henriksson provides the analysis needed to calculate the amount of memory needed and the execution time of the garbage collector.

\subsection{Real-Time GC in the Jamaica JVM}

Siebert 16,17 proposes a combination of scheduling and dividing objects into equally sized blocks in an incremental mark-and-sweep collector. The blocks are garbage collected as separate entities. Arrays are stored either as contiguous memory blocks if such memory can be found fast enough, otherwise arrays are represented as trees.

Siebert also provides an analysis to guarantee non-disruptiveness of the application. The input to the analysis is an upper bound on the amount of reachable memory and the output is the number of GC increments needed per allocated block. 
Table 1. Worst case execution time of operations in real-time garbage collectors (assuming no cyclic garbage.) The size of an object is denoted by $s$ and the minimum amount of free memory (the overhead) by $f$.

\begin{tabular}{llll}
\hline Operation & \multicolumn{2}{l}{ RT-Copying } & RT-Mark-Sweep RTRC \\
\hline Increment (or equivalent) & $O(1)$ & $O(1)$ & $O(1)$ \\
Decrement (or equivalent) & $O(1)$ & $O(1)$ & $O(1)$ \\
Allocation/Free & $O\left(s+\frac{1}{f}\right)$ & $O\left(s+\frac{1}{f}\right)$ & $O(s)$ \\
Member access & $O(1)$ & $O(s)$ & $O(s)$ \\
Array access & $O(1)$ & $O(\log s)$ & $O(\log s)$ \\
\hline
\end{tabular}

\subsection{Comparing to Other Techniques}

As stated above, reference counting has some disadvantages compared to other garbage collection techniques. There do exist real-time copying and mark-sweep collectors, so the need for a real-time reference counting technique might be questioned. Table 1 compares the worst-case execution time of the operations of the real-time reference counter presented in this paper to the worst-case execution times of other real-time garbage collectors [12 17]. Neither the copying nor the mark-sweep algorithm use increment or decrement operations. However, both use read/write barriers that perform equivalent operations. In the table the worst-case execution time of the barriers are compared to the worst-case execution time of the increment/decrement operations. The size of an object is denoted by $s$ and the minimum amount of free memory (the overhead) denoted by $f$.

In real-time systems, the amount of memory is often limited. The real-time reference counting technique enables the usage of all of the memory when no cyclic garbage is produced. If cyclic garbage is produced, the overhead of RTRC is calculated by multiplying the percentage of cyclic garbage with the overhead of a mark-sweep collector, i.e. if $10 \%$ of the garbage belongs to cycles, the overhead is $10 \%$ of the overhead using mark-sweep. Drastically decreasing the memory overhead is especially advantageous for embedded systems.

Copying garbage collectors have the advantage of compacting the memory during garbage collection, which gives fast allocation and eliminates external fragmentation. However, since objects move around, a handle is used to access the object. This cause an extra pointer dereference which could be compared to accessing data in the second block using RTRC. Thus, if most objects can be stored in one or two blocks, accessing members is as fast or faster in RTRC compared to using a copying garbage collector.

\section{Conclusion and Future Work}

This paper presents an automatic memory management technique (RTRC) for hard real-time systems. It does not suffer from external fragmentation and all operations are predictable in worst case memory usage and execution time. All 
(non-cyclic) dead blocks are immediately available for creation of new objects. Thus, the worst case execution time is the same if $10 \%$ or $100 \%$ of the memory is used (assuming no cycles). Even if cyclic garbage is produced, the overhead is drastically decreased for most systems. And finally, the high priority processes can be given the benefit of faster allocation if that is desired.

Previously presented real-time garbage collection techniques that take memory availability into account restricts memory usage. Garbage collectors based on a two sub-heap copying collector can only use half the heap for live objects. Practical experience indicates that often more than five times the live memory is needed to get an acceptable execution time overhead. The memory usage of the mark-sweep collector presented by Siebert [16] is better than that of copying collectors. The figures presented by Siebert suggest that a system need about $50 \%$ memory overhead to get acceptable execution time. The execution time overhead increases rapidly towards infinity when less memory is available.

A backup garbage collector is used to reclaim cyclic data. Having a backup garbage collector might not be a good solution if too much cyclic garbage is produced, but in most systems most cyclic garbage can be avoided. One could use weak pointers (that are not counted,) or one could break cycles manually before they become garbage. A good idea would be to run a backup garbage collector during development of the real-time application as a development aid to find and manually break cycles in the code.

The remaining disadvantage of RTRC is the execution time overhead. The execution time overhead can be greatly reduced using static optimizations. These optimizations can turn heap allocations into stack allocations and remove redundant reference count updates.

The continuation of this research will investigate in more detail topics such as how to optimize performance using static analysis, connect blocks into objects, choose block size, integrating a backup garbage collector, and what to do with large objects.

\section{References}

1. D. Bacon and V. Rajan. Concurrent cycle collection in reference counted systems. In Proceedings of the Fifteenth European Conference on Object-Oriented Programming (ECOOP), Lecture Notes in Computer Science, volume 2072. University Eötvös Loránd, Budapest, Hungary, Springer-Verlag, June 2001.

2. H. G. Baker. Minimising reference count updating with deferred and anchored pointers for functional data structures. ACM SIGPLAN Notices, 29(9), Sept. 1994.

3. J. M. Barth. Shifting garbage collection overhead to compile time. Communications of the ACM, 20(7):513-518, July 1977.

4. D. G. Bobrow. Managing re-entrant structures using reference counts. ACM Transactions on Programming Languages and Systems, 2(3):269-273, July 1980.

5. R. A. Brooks. Trading data space for reduced time and code space in real-time garbage collection on stock hardware. In G. L. Steele, editor, Conference Record of the 1984 ACM Symposium on Lisp and Functional Programming, pages 256-262, Austin, TX, Aug. 1984. ACM Press. 
6. D. R. Brownbridge. Cyclic reference counting for combinator machines. In J.-P. Jouannaud, editor, Record of the 1985 Conference on Functional Programming and Computer Architecture, volume 201 of Lecture Notes in Computer Science, Nancy, France, Sept. 1985. Springer-Verlag.

7. J. M. Bull, L. A. Smith, M. D. Westhead, D. S. Henty, and R. A. Davey. A benchmark suite for high performance Java. Concurrency: Practice and Experience, (12):375-388, 2000.

8. T. W. Christopher. Reference count garbage collection. Software Practice and Experience, 14(6):503-507, June 1984.

9. G. E. Collins. A method for overlapping and erasure of lists. Communications of the ACM, 3(12):655-657, Dec. 1960.

10. L. P. Deutsch and D. G. Bobrow. An efficient incremental automatic garbage collector. Communications of the ACM, 19(9):522-526, Sept. 1976.

11. R. R. Fenichel and J. C. Yochelson. A Lisp garbage collector for virtual memory computer systems. Communications of the ACM, 12(11):611-612, Nov. 1969.

12. R. Henriksson. Scheduling Garbage Collection in Embedded Systems. PhD thesis, Lund Institute of Technology, July 1998.

13. M. S. Johnstone and P. R. Wilson. The memory fragmentation problem: Solved? In P. Dickman and P. R. Wilson, editors, OOPSLA '97' Workshop on Garbage Collection and Memory Management, Oct. 1997.

14. T. Ritzau, M. Beemster, F. Liekweg, and C. Probst. JoC - the JOSES compiler. Presented at the Java for Embedded Systems Workshop, London, May 2000.

15. R. A. Saunders. The LISP system for the Q-32 computer. In E. C. Berkeley and D. G. Bobrow, editors, The Programming Language LISP: Its Operation and Applications, pages 220-231, Cambridge, MA, 1974. Information International, Inc.

16. F. Siebert. Guaranteeing non-disruptiveness and real-time deadlines in an incremental garbage collector. In R. Jones, editor, Proceedings of the First International Symposium on Memory Management, volume 34(3) of ACM SIGPLAN Notices, pages 130-137, Vancouver, Oct. 1998. ACM Press. ISMM is the successor to the IWMM series of workshops.

17. F. Siebert. Eliminating external fragmentation in a non-moving garbage collector for Java. In Proceedings of Compilers, Architectures and Synthesis for Embedded Systems (CASES'00), San Jose, November 2000.

18. A. Veen. The JOSES project - compiling Java for embedded systems. In D. U. Assmann, editor, Java Optimization Strategies for Embedded Systems Workshop at ETAPS 2001, Genova, Italy, Apr. 2001.

19. J. Weizenbaum. Symmetric list processor. Communications of the ACM, 6(9):524544, Sept. 1963. 